Article

\title{
Fully Differential Chopper-Stabilized Multipath Current-Feedback Instrumentation Amplifier with R-2R DAC Offset Adjustment for Resistive Bridge Sensors
}

\author{
Yongsu Kwon ${ }^{1}$, Hyungseup Kim ${ }^{1}{ }^{1}$, Jaesung Kim ${ }^{1}$, Kwonsang Han ${ }^{1}$, Donggeun You ${ }^{1}$, \\ Hyunwoo Heo ${ }^{1}$, Dong-il "Dan" Cho ${ }^{2}$ and Hyoungho Ko ${ }^{1, *(\mathbb{D}}$ \\ 1 Department of Electronics Engineering, Chungnam National University, Daejeon 34134, Korea; \\ yongmayer@cnu.ac.kr (Y.K.); hyungseup@cnu.ac.kr (H.K.); jskim1@cnu.ac.kr (J.K.); kshan1@cnu.ac.kr (K.H.); \\ ryad142@cnu.ac.kr (D.Y.); hwheo@o.cnu.ac.kr (H.H.) \\ 2 ASRI/ISRC, Department of Electrical and Computer Engineering, Seoul National University, Seoul 08826, \\ Korea; dicho@snu.ac.kr \\ * Correspondence: hhko@cnu.ac.kr; Tel.: +82-42-821-5664
}

Received: 2 December 2019; Accepted: 18 December 2019; Published: 20 December 2019

\begin{abstract}
A fully differential multipath current-feedback instrumentation amplifier (CFIA) for a resistive bridge sensor readout integrated circuit (IC) is proposed. To reduce the CFIA's own offset and $1 / \mathrm{f}$ noise, a chopper stabilization technique is implemented. To attenuate the output ripple caused by chopper up-modulation, a ripple reduction loop (RRL) is employed. A multipath architecture is implemented to compensate for the notch in the chopping frequency band of the transfer function. To prevent performance degradation resulting from external offset, a 12-bit R-2R digital-to-analog converter (DAC) is employed. The proposed CFIA has an adjustable gain of $16-44 \mathrm{~dB}$ with 5-bit programmable resistors. The proposed resistive sensor readout IC is implemented in a $0.18 \mu \mathrm{m}$ complementary metal-oxide-semiconductor (CMOS) process. The CFIA draws $169 \mu \mathrm{A}$ currents from a $3.3 \mathrm{~V}$ supply. The simulated input-referred noise and noise efficiency factor (NEF) are $28.3 \mathrm{nV} / \sqrt{\mathrm{Hz}}$ and 14.2, respectively. The simulated common-mode rejection ratio (CMRR) is $162 \mathrm{~dB}$, and the power supply rejection ratio (PSRR) is $112 \mathrm{~dB}$.
\end{abstract}

Keywords: multipath current-feedback instrumentation amplifier (CFIA); resistive bridge sensor readout integrated circuit (IC); chopper stabilization technique; ripple reduction loop (RRL)

\section{Introduction}

Recently, the Internet of Things (IoT) has played a significant role in many aspects of modern life, such as medical care, automobiles, homes, and amenities [1]. IoT sensors that perceive and collect information and circuits that readout these sensors with high precision are also becoming more important [2]. Resistive micro-electro-mechanical systems (MEMS) sensors that use a piezoresistive effect have various ways, such as strain gauges and pressure, acceleration, and force sensors [3-7]. The need for smaller, less expensive high-performance sensors has developed in a way that makes sensors and sensing elements smaller. To readout these sensors precisely, readout circuits should have low-noise, high input impedance, low power, and high common-mode rejection ratio (CMRR) characteristics. Conventionally, the well-known 3-opamp instrumentation amplifier (IA) scheme features a high input impedance. Its disadvantage is that the degree of matching resistance greatly influences the CMRR, making it difficult to achieve a high CMRR. A current-feedback instrumentation amplifier (CFIA) consists of an input transconductance stage and output Miller integrator stage. 
The CFIA scheme features high input impedance, high CMRR, high open-loop gain, and low power consumption, because the input common-mode $(\mathrm{CM})$ voltage is isolated by the input transconductance stage [8]. Although the CFIA has advantages in terms of its simple structure, the input offset and noise can cause performance degradation. To implement the low-noise characteristics of the instrumentation amplifier (IA), auto-zeroing, correlated double sampling (CDS), and the chopper stabilization technique are the most widely used schemes [9-12]. Unlike thermal noise with a wide frequency band, flicker noise and DC offset overlap with the sensor output signal. For the desired output voltage of the IA, reduction of flicker noise and DC offset are essential. The auto-zeroing and CDS schemes sample the offsets in the input capacitor of the IA, which attenuates flicker noise and DC offset through comparison and amplification. However, the high-frequency thermal noise folds and appears in integrated form in the baseband because of aliasing. The chopper stabilization technique reduces baseband noise through up-modulating the flicker noise and DC offset in the baseband; thus, a thermal-noise-limited level can be achieved. However, up-modulated DC offset and flicker noise can cause a ripple in the output. To solve this problem, a ripple reduction loop (RRL) is used to demodulate the ripple and provide negative feedback to the input through the integrator [13]. The RRL attenuates the output ripple caused by chopping, but there are disadvantages to generating notches of the transfer function in the chopping frequency band.

In this study, a low-noise bridge resistive sensor readout circuit with dual offset reduction is implemented. (1) The offset inside the amplifier is reduced by the multipath offset stabilization topology. (2) The offset outside the amplifier is adjusted by the R-2R digital-to-analog converter (DAC) in the current-feedback path. To attenuate the flicker noise and DC offset in the baseband, the chopper stabilization technique is employed. The RRL is adopted to attenuate the output ripple. A multipath topology is employed to compensate for the notches of the transfer function in the chopping frequency band. This CFIA achieves a high CMRR, high input impedance, and low power consumption.

\section{Circuit Implementation}

Figure 1 shows the proposed resistive sensor readout analog-front-end (AFE) IC architecture. The architecture consists of a multipath CFIA, low-pass filter (LPF), buffer, 12-bit successive approximation register analog-to-digital converter (SAR ADC), R-2R DAC, and feedback resistor. To drive a variety of resistive sensors, the feedback resistor $R_{2}$ is designed by implementing a 5-bit programmable resistor. Depending on the external 5-bit digital input, the proposed resistive sensor readout AFE has an adjustable gain of 16-44 dB. Figure 2 shows the operation of the R-2R DAC calibration. The R-2R DAC is designed to compensate for the external offset generated by sensor element mismatches. The R-2R DAC has the ability to adjust the amplified external offset voltage between $3.3 \mathrm{~V}$ and $-3.3 \mathrm{~V}$, with $1.61 \mathrm{mV}$ intervals through the 12-bit digital input. The output voltage of the proposed CFIA without the external offset and R-2R DAC is expressed as (1).

$$
\text { OUTP }- \text { OUTN }=\left(1+2 \times \frac{R_{1}}{R_{2}}\right) \times(I N P-I N N)
$$

Each unit of the R-2R DAC, consisting of $R_{D}$ and $2 R_{D}$ resistors, has an output resistance of $R_{D}$. The output resistance between the DACP and DACN node can be interpreted as $2 R_{D}$. Therefore, the output voltage of the proposed CFIA with R-2R DAC is expressed as (2).

$$
\begin{aligned}
\text { OUTP }- \text { OUTN }= & \left(1+2 \times \frac{R_{1}}{R_{2}}\right) \times(I N P-I N N) \\
& +\frac{R_{1}}{R_{D}} \times((I N P-I N N)-(D A C P-D A C N))
\end{aligned}
$$


Through the second term of (2), the amplified external offset can be calibrated. In this design, $\mathrm{R}_{1}$ can influence offset calibration. Therefore, $R_{2}$, which is independent from offset calibration, is configured as a programmable resistor.

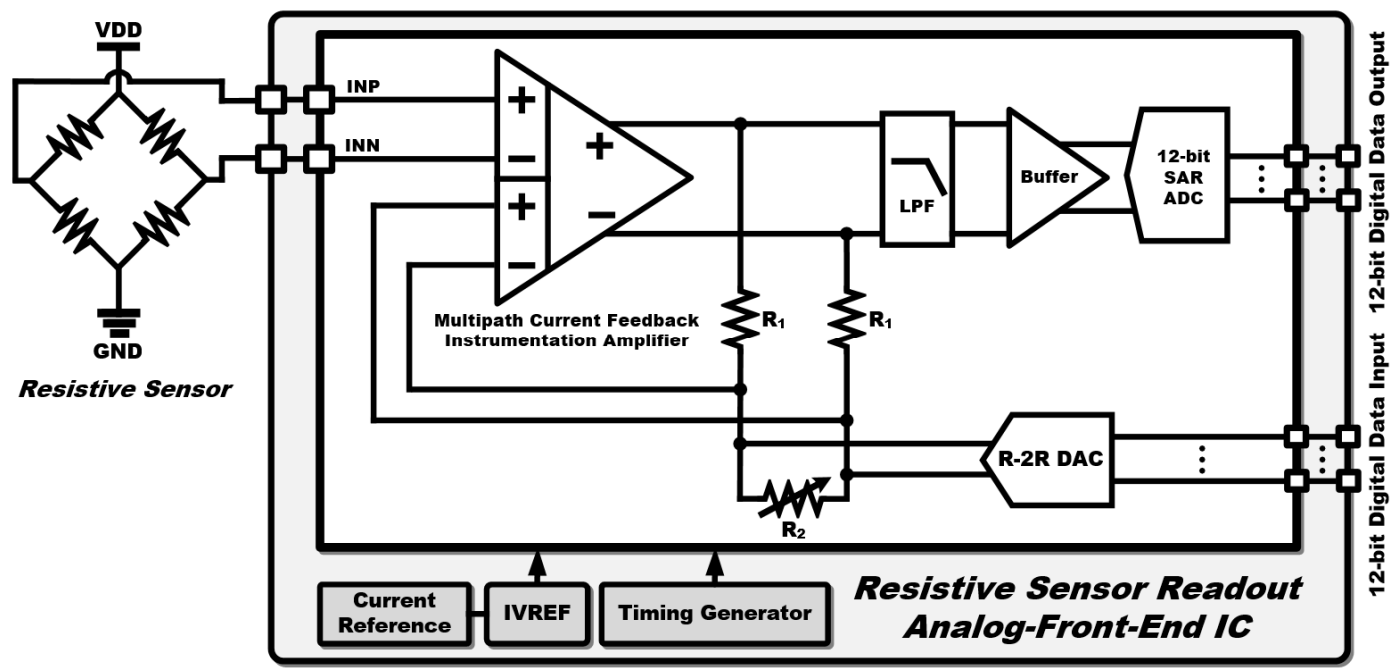

Figure 1. Proposed architecture of resistive sensor readout analog-front-end integrated circuit (IC).

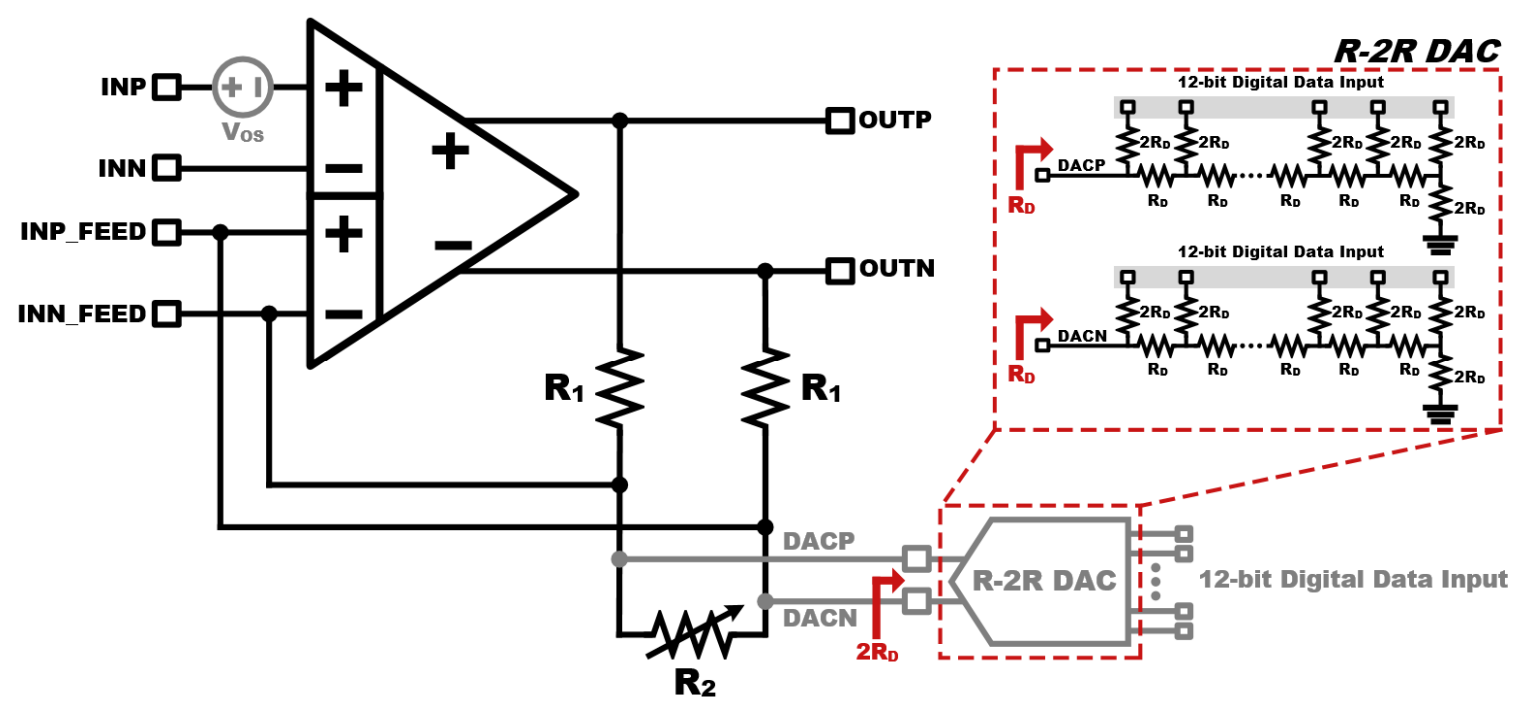

Figure 2. Operation of R-2R digital-to-analog converter (DAC) calibration.

Figure 3 shows the block diagram of the proposed CFIA. The proposed fully differential CFIA circuit consists of two main signal paths: a low-frequency and a high-frequency path. The low-frequency path employs the chopper stabilization technique to reduce flicker noise and DC offset in the baseband. To reduce the ripple caused by chopping, the RRL is implemented. To compensate for the notch in the transfer function of the chopping frequency band, the high-frequency path is used. 


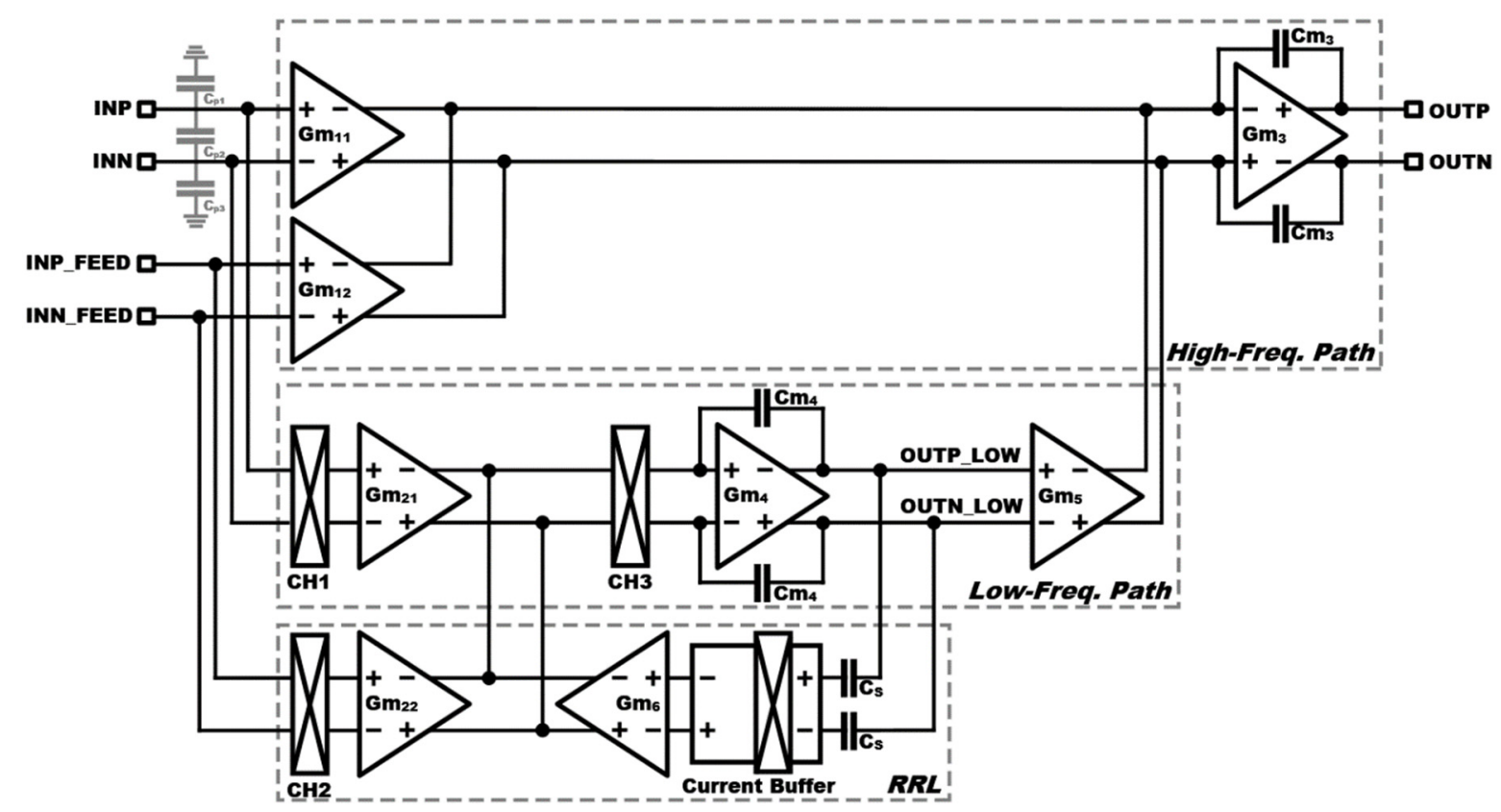

Figure 3. Block diagram of the proposed current-feedback instrumentation amplifier (CFIA).

\subsection{Low-Frequency Path}

The R-2R DAC reduces the offset caused by sensor element mismatches but does not reduce the IA's own internal offset. The chopper implements an internal offset reduction. The chopper is operated by two nonoverlapping clocks. As shown in Figure 3, the sensor output signal is modulated into the high-frequency band through a modulation chopper ( $\mathrm{CH} 1)$, and the modulated signal is demodulated back into the baseband through the demodulation chopper (CH3). Simultaneously, a modulation chopper $(\mathrm{CH} 3)$ modulates flicker noise and DC offset into the high-frequency band. Consequentially, the up-modulated flicker noise and DC offset are reduced through a Miller integrator $\left(\mathrm{Gm}_{4}\right)$ and combined with high-frequency path signals. At the output of the Miller integrator $\left(\mathrm{Gm}_{4}\right)$, the up-modulated flicker noise and DC offset generate a ripple. The ripple is negative feedback to the input of the chopper $(\mathrm{CH} 3)$ through an $\mathrm{AC}$ coupling capacitor $\left(\mathrm{C}_{\mathrm{s}}\right)$ and the current buffer.

The overall input impedance of the CFIA is determined by the switched capacitor resistor, and is generated by the chopping frequency and input parasitic capacitor $\left(C_{p 1}, C_{p 2}\right.$, and $\left.C_{p 3}\right)$. Consequently, the input impedance is inversely proportional to the chopping frequency and input parasitic capacitor. If the chopping frequency is reduced to increase the impedance, the output ripple increases. If the parasitic capacitor is reduced to increase the impedance, flicker noise tends to prevail. Achieving high-performance CFIA requires delicate consideration of trade-offs between noise and input impedance.

\subsection{High-Frequency Path}

Figure 4 shows a schematic of the high-frequency path. The high-frequency path consists of a three-input transconductance stage and the Miller integrator. The input, feedback, and low-frequency path output signals are converted to currents through $\mathrm{Gm}_{11}, \mathrm{Gm}_{12}$, and $\mathrm{Gm}_{5}$, respectively, and entered into the Miller integrator. At the $\mathrm{Gm}_{3}$ stage, to achieve a fine-frequency response in the high-frequency path, nested Miller compensation is implemented [14]. To compensate for the drawbacks of the dead-band in the crossover area, a Monticelli class AB output stage is implemented (M17, M18, M19, and M20). It has the advantages of linear output characteristics and distortion reduction. Figure 5 shows a schematic of the high-frequency path common-mode feedback (CMFB) circuit. The gate voltage of transistors M13 and M14 is determined by the active CMFB circuit, which has the advantage 
of increasing bandwidth. To stabilize the output voltage, the CMFB circuit is also implemented at the output stage.

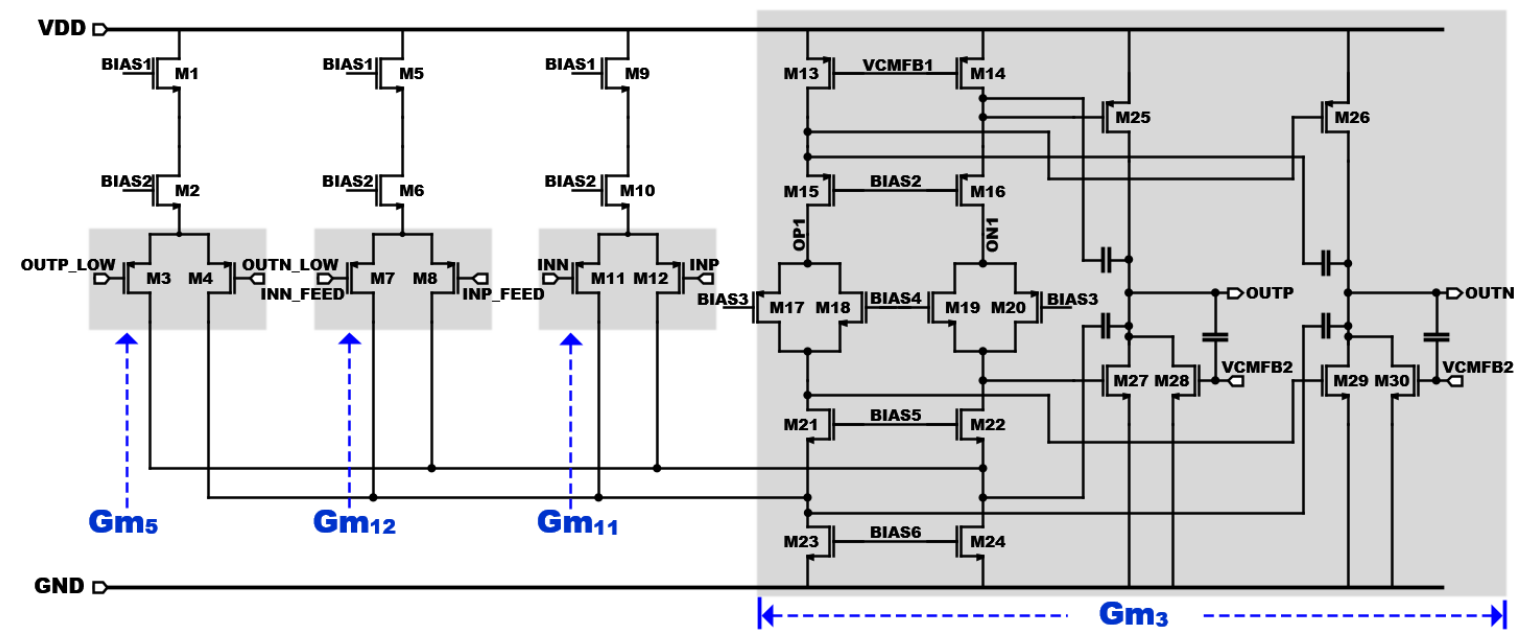

Figure 4. Schematic of the high-frequency path.

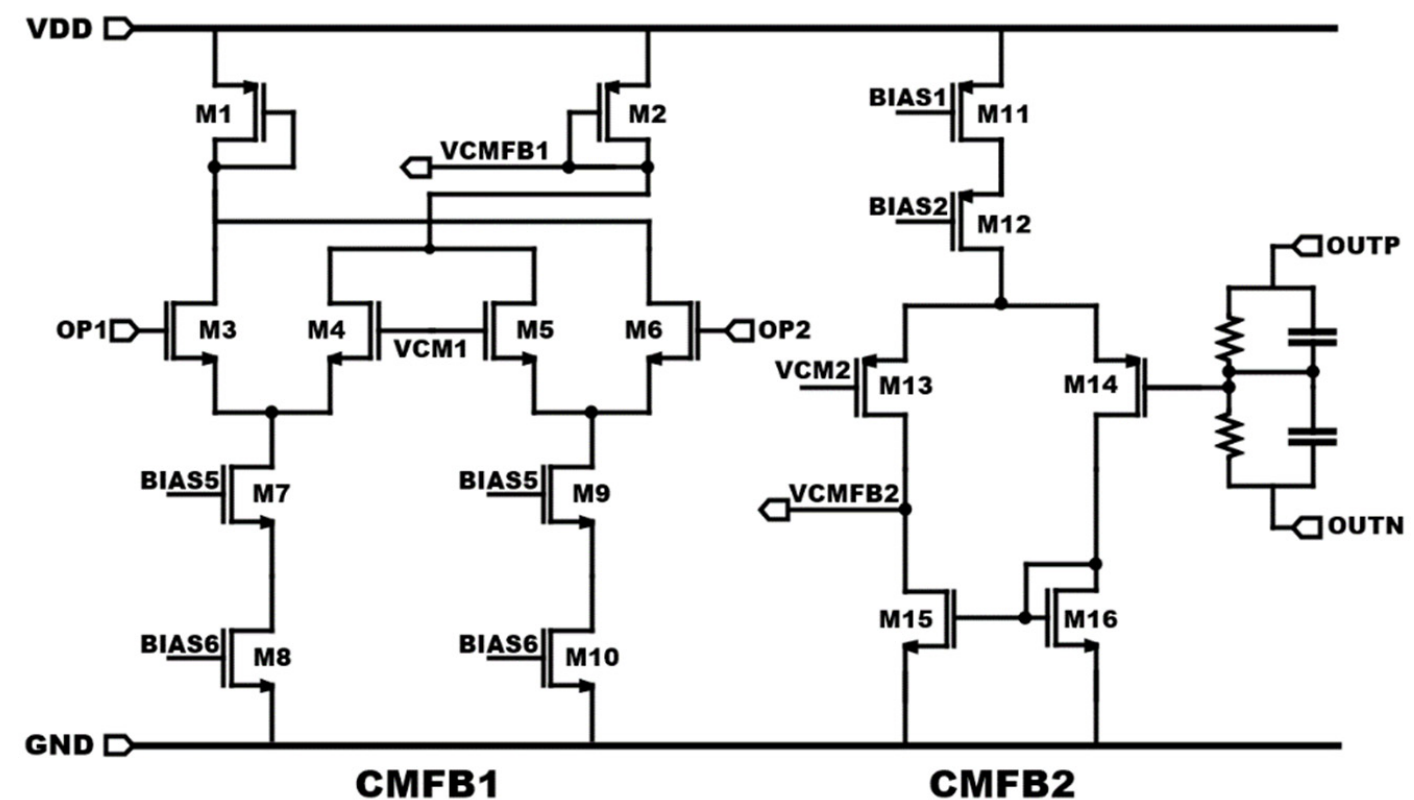

Figure 5. Schematic of the high-frequency path common-mode feedback (CMFB) circuit.

\section{Simulation Results}

Figure 6 shows the transient response of a CFIA with a $20 \mathrm{mV}$ amplitude input sine wave of $1 \mathrm{kHz}$. The gain of the CFIA was set to have $11 \mathrm{~V} / \mathrm{V}$ through a 5-bit programmable resistor. The graph shows the input/output in the form of a differential signal. The output signal is simulated to have a peak value of $220.07 \mathrm{mV}$ at $0.25 \mathrm{~ms}$. Figure 7 shows the simulated transfer function in differential mode, VDD, GND, and common-mode. The simulated CMRR achieves $162 \mathrm{~dB}$. The power supply rejection ratio (PSRR+ and PSRR-) are simulated at 112 and $110 \mathrm{~dB}$, respectively. Figure 8 shows the frequency response of the CFIA. The open-loop gain is simulated at $132.5 \mathrm{~dB}$ at $1 \mathrm{mHz}$. The simulated unit gain bandwidth (UGBW) is $59.2 \mathrm{kHz}$, and the phase margin is $75.1^{\circ}$ at UGBW. Figure 9 shows the simulated input-referred noise. The simulated input-referred noise is $30 \mathrm{nV} / \sqrt{ } \mathrm{Hz}$ at $1 \mathrm{~Hz}$ and $28.3 \mathrm{nV} / \sqrt{\mathrm{Hz}}$ at $1 \mathrm{kHz}$. 


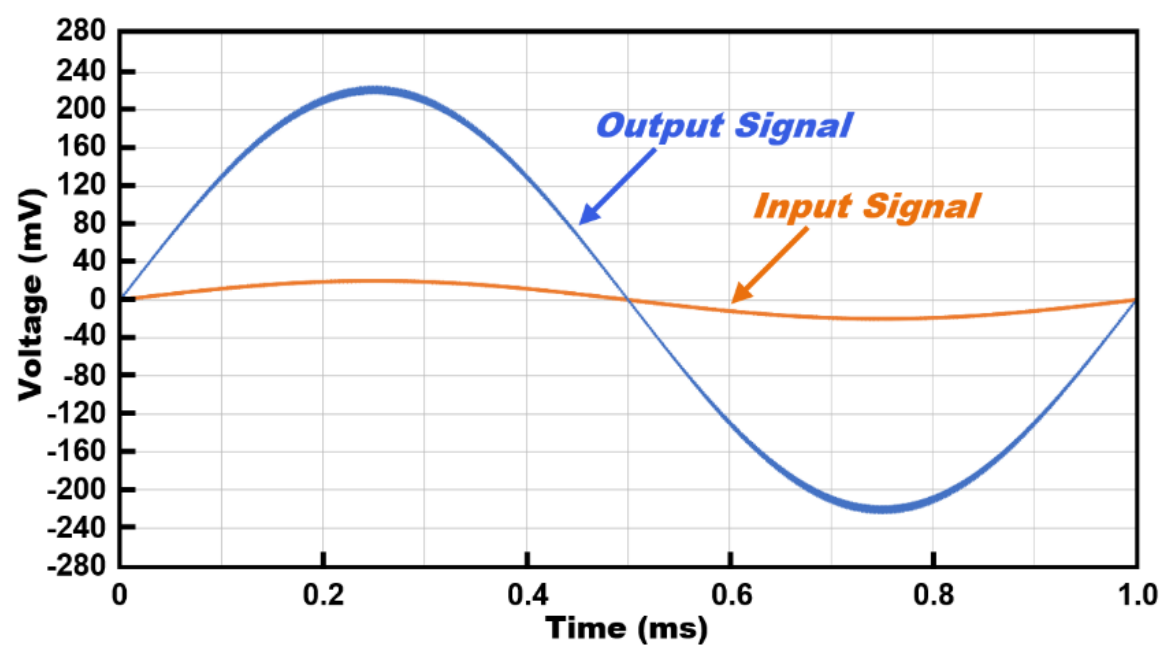

Figure 6. Transient response of CFIA with $20 \mathrm{mV}$ amplitude input sine wave.

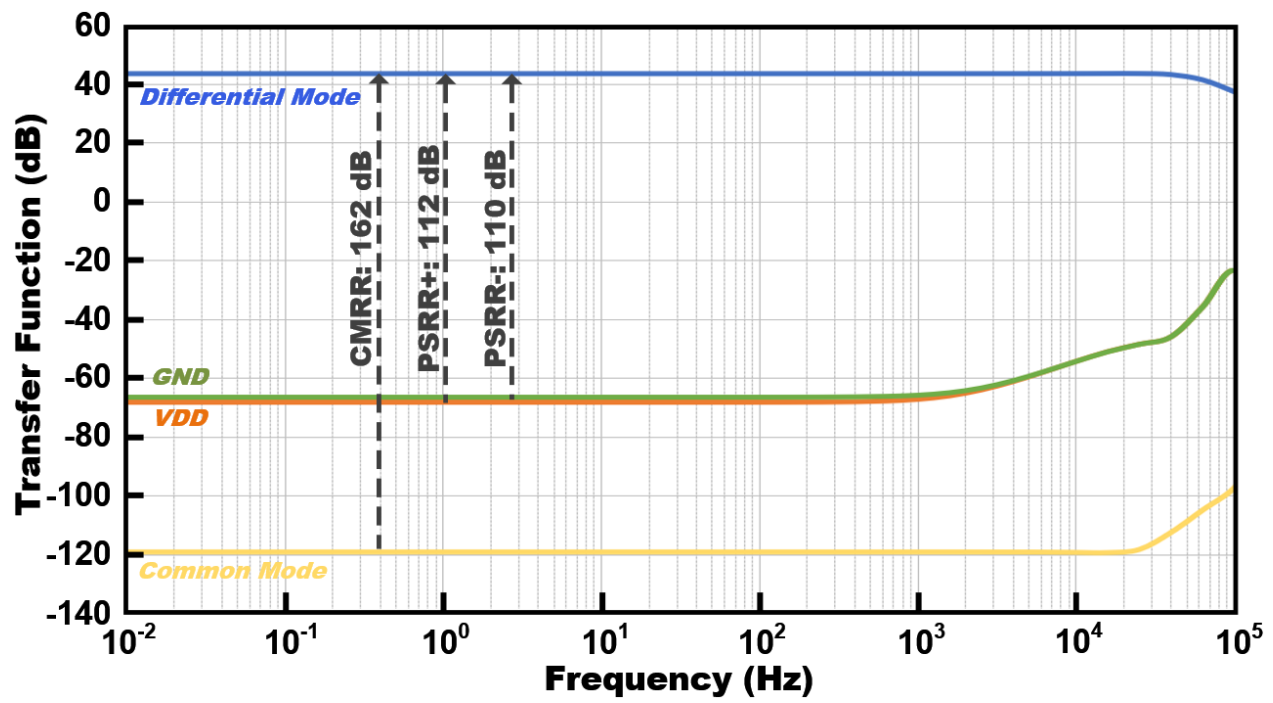

Figure 7. Simulated transfer function with differential mode, VDD, ground (GND), and common-mode.

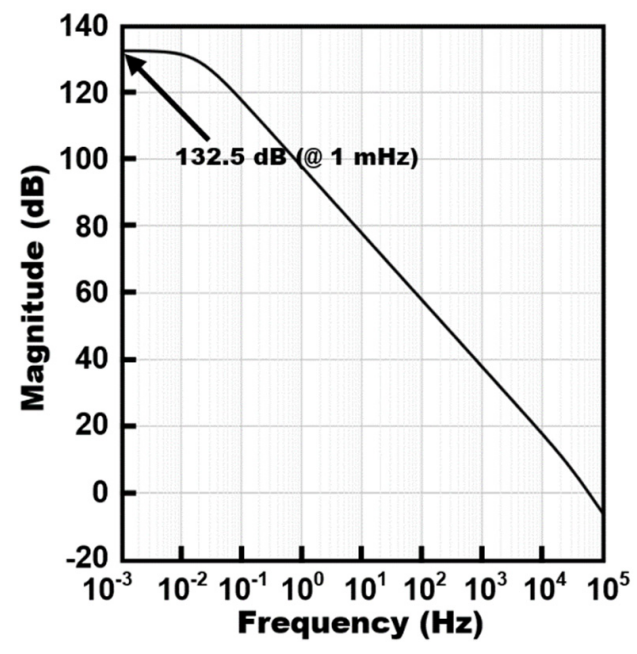

(a)

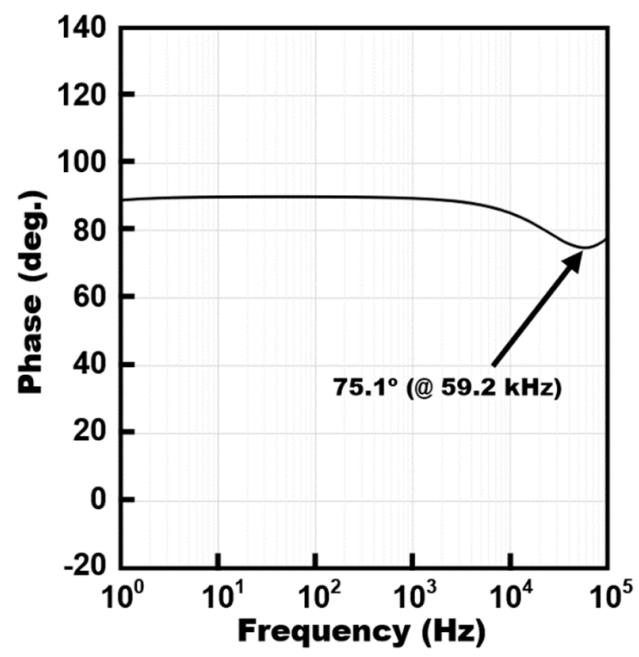

(b)

Figure 8. (a) Gain of the CFIA and (b) phase of the CFIA. 


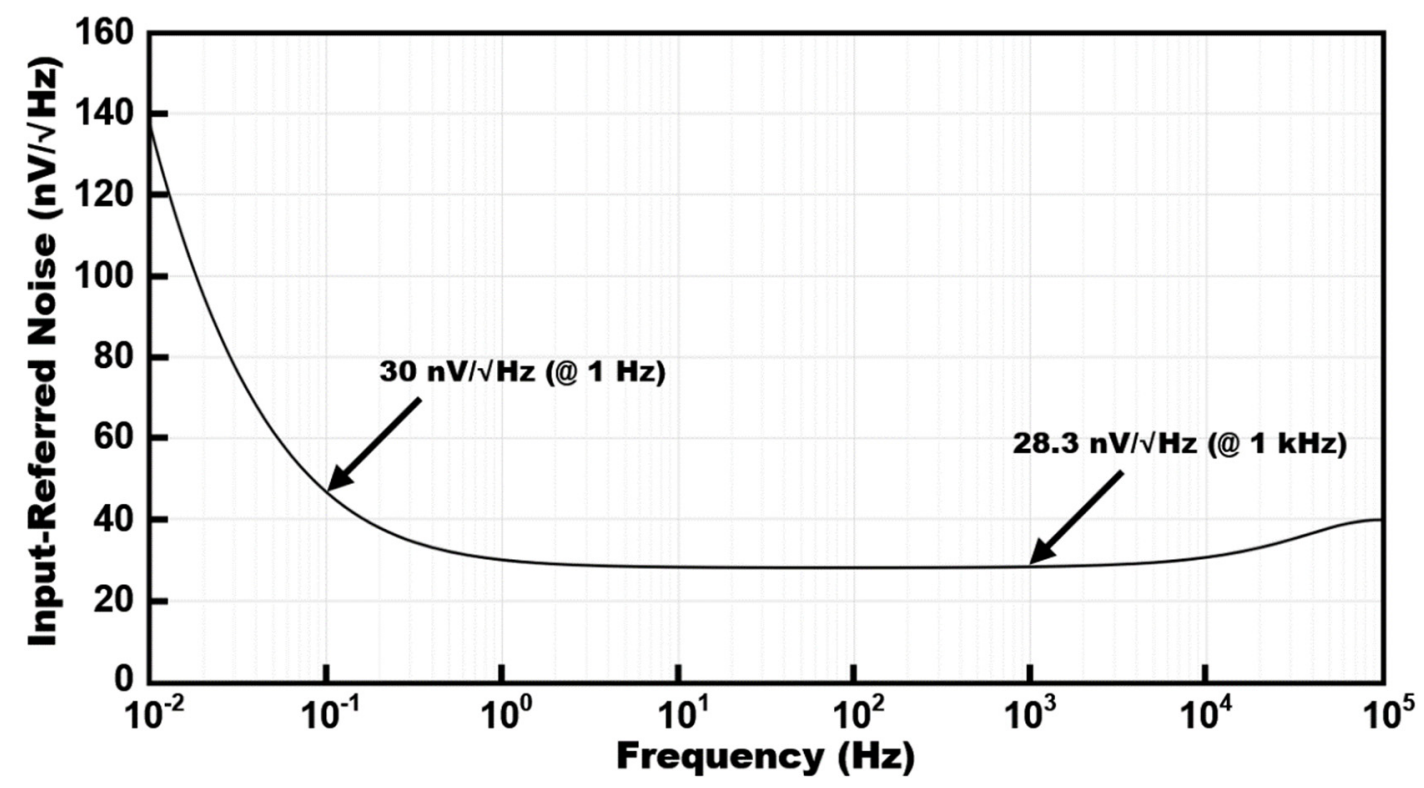

Figure 9. Simulated input-referred noise of CFIA.

Figure 10 shows the simulated CFIA output DC operating point under R-2R DAC digital input variation. It shows that the offset of the CFIA output can be linearly calibrated within a $\pm 3.3 \mathrm{~V}$ range. The performance summary and comparison with other papers are shown in Table 1 . The efficiency of the instrumentation amplifier can be evaluated using a noise efficiency factor (NEF), as shown in (3), where $V_{r m s, n i}$ is the input-referred noise RMS value, $I_{t o t}$ is the total current consumption, $\mathrm{U}_{\mathrm{T}}$ is thermal voltage, and BW is bandwidth of the amplifier.

$$
N E F=V_{r m s, n i} \times \sqrt{\frac{2 \times I_{\text {tot }}}{\pi \times U_{T} \times 4 k T \times B W}}
$$

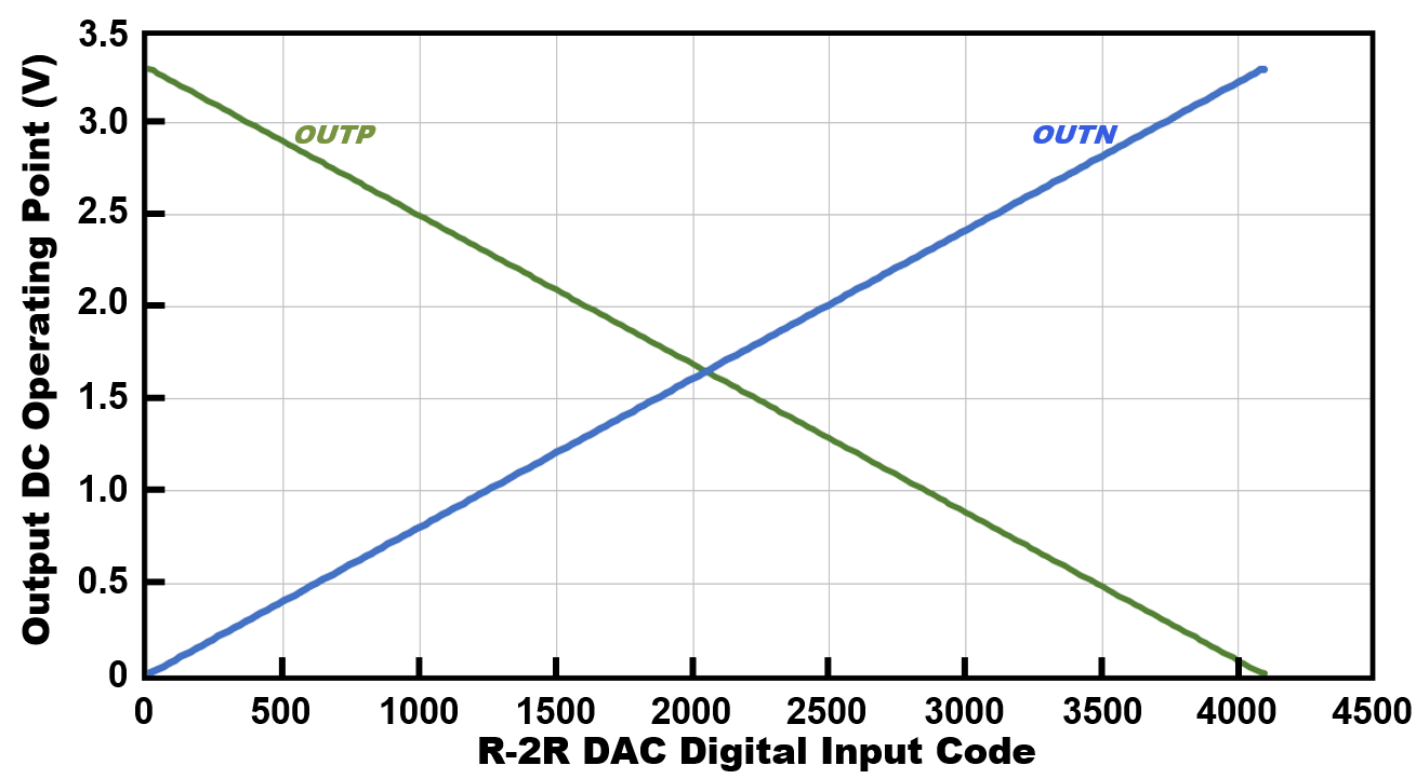

Figure 10. Simulated CFIA output DC operating point under R-2R DAC digital input variation. 
Table 1. Performance summary of proposed current-feedback instrumentation amplifier (CFIA) and comparison with other instrumentation amplifiers (IAs).

\begin{tabular}{|c|c|c|c|c|c|}
\hline & This Work & [9] & [15] & [16] & [17] \\
\hline Process $(\mu \mathrm{m})$ & 0.18 & 0.5 & 0.7 & 0.7 & 0.32 \\
\hline Supply (V) & 3.3 & $3-5.5$ & 5 & 5.5 & 3.3 \\
\hline Current $(\mu \mathrm{A})$ & 169 & 1700 & 290 & 325 & 170 \\
\hline DC gain $(\mathrm{dB})$ & 132.5 & 1000 & - & - & 201.2 \\
\hline CMRR (dB) & 162 & 142 & 127 & 130 & $>120$ \\
\hline PSRR $+(\mathrm{dB})$ & 112 & 138 & 130 & 114 & 115 \\
\hline PSRR $-(\mathrm{dB})$ & 110 & - & - & - & - \\
\hline $\mathrm{BW}(\mathrm{kHz})$ & 59.2 & 800 & 800 & 640 & 40 \\
\hline $\begin{array}{l}\text { Input-referred noise } \\
(\mathrm{nV} / \sqrt{ } \mathrm{Hz})\end{array}$ & 28.3 & 27 & 17 & 42 & 18 \\
\hline NEF & 14.2 & 43 & 11.2 & 29.2 & 10.6 \\
\hline
\end{tabular}

\section{Discussion and Conclusions}

A fully differential multipath CFIA for a bridge resistive sensor readout IC is presented. The proposed CFIA has the ability to reduce external and internal offsets, thereby achieving low-noise characteristics. To attenuate the internal offset, the chopper stabilization technique is implemented. To reduce the output ripple caused by chopper up-modulation, an RRL is used. A multipath architecture is employed to compensate for the notch in the chopping frequency band of the transfer function. To calibrate external offset, a 12-bit R-2R DAC is adopted. The proposed resistive sensor readout IC is implemented using $0.18 \mu \mathrm{m}$ CMOS technology. The current consumption of CFIA is $169 \mu \mathrm{A}$ with a $3.3 \mathrm{~V}$ supply. The simulated input-referred noise and NEF are $28.3 \mathrm{nV} / \sqrt{ } \mathrm{Hz}$ and 14.2 , respectively. The CFIA achieves $112 \mathrm{~dB}$ PSRR+ and $162 \mathrm{~dB}$ CMRR. The proposed CFIA, which achieves low-noise characteristics, is expected to be employed in various resistive IoT sensor applications with high precision.

Author Contributions: Conceptualization and supervision, H.K.; investigation and writing-original draft preparation, Y.K.; and writing-review and editing, H.K., J.K., K.H., D.Y., H.H., and D.-i.D.C. All authors have read and agreed to the published version of the manuscript.

Funding: This work was supported by a grant to the Bio-Mimetic Robot Research Center Funded by Defense Acquisition Program Administration and by the Agency for Defense Development (UD160027ID).

Acknowledgments: The EDA tool was supported by the IC Design Education Center (IDEC), Korea.

Conflicts of Interest: The authors declare no conflict of interest.

\section{References}

1. Vashi, S.; Ram, J.; Modi, J.; Verma, S.; Prakash, C. Internet of Things (IoT): A vision, architectural elements, and security issues. In Proceedings of the IoT in Social, Mobile, Analytics and Cloud (I-SMAC), Palladam, India, 10-11 February 2017; pp. 492-496. [CrossRef]

2. Al-Fuqaha, A.; Guizani, M.; Mohammadi, M.; Aledhari, M.; Ayyash, M. Internet of Things: A Survey on Enabling Technologies, Protocols, and Applications. IEEE Commun. Surv. Tutor. 2015, 17, 2347-2376. [CrossRef]

3. Hebrard, L.; Kammerer, J.B.; Braun, F. A chopper stabilized biasing circuit suitable for cascaded wheatstone-bridge-like sensors. IEEE Trans. Circuits Syst. I Regul. Pap. 2005, 52, 1653-1665. [CrossRef]

4. Barlian, A.A.; Park, W.; Mallon, J.R.; Rastegar, A.J.; Pruitt, B.L. Review: Semiconductor Piezoresistance for Microsystems. Proc. IEEE 2009, 97, 513-552. [CrossRef] [PubMed]

5. Gowrishetty, U.; Walsh, K.; McNamara, S.; Roussel, T.; Aebersold, J. Single element 3-terminal pressure sensors: A new approach to pressure sensing and its comparison to the half bridge sensors. In Proceedings of the Solid-State Sensors, Actuators and Microsystems, Denver, CO, USA, 21-25 June 2009; pp. 1134-1137. [CrossRef] 
6. Gao, S.; Yi, Z.; Ye, Y.; Qin, M.; Huang, Q. Configuration of a Self-Heated Double Wheatstone Bridge for 2-D Wind Sensors. J. Microelectromech. Syst. 2019, 28, 125-130. [CrossRef]

7. Wei, J.; Porta, M.; Tichem, M.; Staufer, U.; Sarro, P.M. Integrated Piezoresistive Force and Position Detection Sensors for Micro-Handling Applications. J. Microelectromech. Syst. 2013, 22, 1310-1326. [CrossRef]

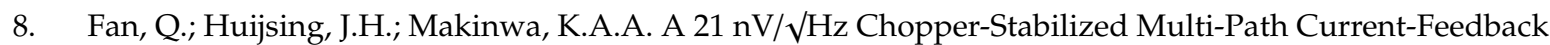
Instrumentation Amplifier with $2 \mu \mathrm{V}$ Offset. IEEE J. Solid State Circuits 2012, 47, 464-475. [CrossRef]

9. Pertijs, M.A.P.; Kindt, W.J. A 140 dB-CMRR Current-Feedback Instrumentation Amplifier Employing Ping-Pong Auto-Zeroing and Chopping. IEEE J. Solid State Circuits 2010, 45, 2044-2056. [CrossRef]

10. Oliaei, O. Noise analysis of correlated double sampling SC integrators with a hold capacitor. IEEE Trans. Circuits Syst. I Fundam. Theory Appl. 2003, 50, 1198-1202. [CrossRef]

11. Lee, C.; Song, J. A Chopper Stabilized Current-Feedback Instrumentation Amplifier for EEG Acquisition Applications. IEEE Access 2019, 7, 11565-11569. [CrossRef]

12. Enz, C.C.; Temes, G.C. Circuit techniques for reducing the effects of op-amp imperfections: Autozeroing, correlated double sampling, and chopper stabilization. Proc. IEEE 1996, 84, 1584-1614. [CrossRef]

13. Wu, R.; Makinwa, K.A.A.; Huijsing, J.H. A Chopper Current-Feedback Instrumentation Amplifier With a $1 \mathrm{mHz}$ 1/f Noise Corner and an AC-Coupled Ripple Reduction Loop. IEEE J. Solid State Circuits 2009, 44, 3232-3243. [CrossRef]

14. Eschauzier, R.G.H.; Hogervorst, R.; Huijsing, J.H. A programmable 1.5 V CMOS class-AB operational amplifier with hybrid nested Miller compensation for $120 \mathrm{~dB}$ gain and $6 \mathrm{MHz}$ UGF. IEEE J. Solid State Circuits 1994, 29, 1497-1504. [CrossRef]

15. Wu, R.; Huijsing, J.H.; Makinwa, K.A.A. A Current-Feedback Instrumentation Amplifier with a Gain Error Reduction Loop and 0.06\% Untrimmed Gain Error. IEEE J. Solid State Circuits 2011, 46, 2794-2806. [CrossRef]

16. Witte, J.F.; Huijsing, J.H.; Makinwa, K.A.A. A chopper and auto-zero offset-stabilized CMOS instrumentation amplifier. In Proceedings of the VLSI Circuits, Kyoto, Japan, 16-18 June 2009; pp. 210-211.

17. Butti, F.; Piotto, M.; Bruschi, P. A Chopper Instrumentation Amplifier with Input Resistance Boosting by Means of Synchronous Dynamic Element Matching. IEEE Trans. Circuits Syst. I Regul. Pap. 2017, 64, 753-764. [CrossRef]

(C) 2019 by the authors. Licensee MDPI, Basel, Switzerland. This article is an open access article distributed under the terms and conditions of the Creative Commons Attribution (CC BY) license (http://creativecommons.org/licenses/by/4.0/). 